\title{
Predictors of response to exercise training in patients with coronary artery disease - a subanalysis of the SAINTEX-CAD study
}

Isabel Witvrouwen ${ }^{1}$, Nele Pattyn ${ }^{2}$, Andreas B Gevaert ${ }^{1}$, Nadine Possemiers ${ }^{3}$, Amaryllis H Van Craenenbroeck ${ }^{4}$, Veronique Cornelissen ${ }^{2}$, Luc Vanhees ${ }^{2}$, Paul J Beckers ${ }^{3}$, Emeline M Van Craenenbroeck ${ }^{1,3}$

${ }^{1}$ Laboratory of Cellular and Molecular Cardiology, Department of Cardiology, Antwerp University Hospital, Edegem, Belgium

${ }^{2}$ Department of Rehabilitation Sciences, Research Centre for Cardiovascular and Respiratory Rehabilitation, University of Leuven, Leuven, Belgium

${ }^{3}$ Cardiac Rehabilitation Centre, Department of Cardiology, Antwerp University Hospital, Edegem, Belgium

${ }^{4}$ Laboratory of Experimental Medicine and Paediatrics, Antwerp University Hospital, Edegem, Belgium

Corresponding author and reprint requests:

Isabel Witvrouwen

Laboratory of Cellular and Molecular Cardiology, Department of Cardiology, Antwerp University Hospital

Wilrijkstraat 10, 2650 Edegem, Belgium

+3238214098

isabel.witvrouwen@uantwerpen.be

IW and EMVC are supported by the Fund for Scientific Research Flanders with a predoctoral fellowship (1194918N) and senior clinical investigator fellowship (1804315N), respectively.

Information about previous presentations:

The abstract "Predictors of response to exercise training in patients with coronary artery disease" was winner of the young investigator award at Europrevent 2018 session III Basic \& Translational Research, presented by Isabel Witvrouwen.

No conflict of interest.

Word count: 893 (excl. abstract and references) - Max. 800 


\begin{abstract}
Exercise training (ET) improves peak oxygen uptake ( $\mathrm{VO}_{2}$ peak), an important predictor of mortality in coronary artery disease (CAD) patients. The influence of clinical and disease characteristics on training response is not well established in CAD. Therefore, we aimed to evaluate whether baseline cardiovascular disease variables and training intensity can predict the maximal aerobic response to ET. The Study on Aerobic INTerval EXercise training in CAD patients (SAINTEX-CAD) previously showed that 12 weeks of aerobic interval training and continuous training equally improved $\mathrm{VO}_{2}$ peak in CAD patients. We identified 24 exercise non-responders (ENR, change $\mathrm{VO}_{2}$ peak $<1 \mathrm{ml} / \mathrm{kg} / \mathrm{min}$ ) among 167 participants to SAINTEXCAD. In a between-group comparison, ENR were older, their baseline $\mathrm{VO}_{2}$ peak and oxygen uptake efficiency slope (OUES) were higher, and ENR were more frequently included after elective percutaneous coronary intervention $(\mathrm{PCl})($ all $p<0.05)$. In a logistic regression analysis, age (odds ratio $(O R)=1.11(1.04-1.18), p=0.001)$, history of elective $\mathrm{PCl}(\mathrm{OR}=3.31$ (1.12-9.76), $p=0.030$ ) and higher baseline $\mathrm{VO}_{2}$ peak (OR=1.16 (1.06-1.27), $p=0.001$ ) were independent predictors of exercise non-response. In a linear regression analysis, age $(\beta=-$

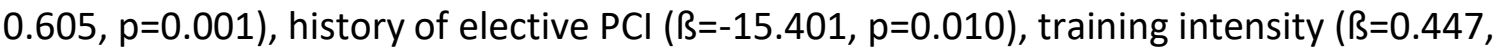
$p=0.008)$, baseline physical activity $(\beta=0.014, p=0.003)$ and OUES $(\beta=-0.014, p<0.001)$ independently predicted change in $\mathrm{VO}_{2}$ peak and explained $41 \%$ of the variability in change in $\mathrm{VO}_{2}$ peak.

To summarize, $14 \%$ of CAD patients were ENR. Higher baseline $\mathrm{VO}_{2}$ peak and OUES, history of elective $\mathrm{PCl}$, older age, lower training intensity and lower baseline physical activity were independent predictors of training non-response. Identification of patients with a large likelihood of non-response is a first step towards patient tailored exercise programmes.
\end{abstract}

\title{
Abstract word count: 256
}

\section{Keywords}

Coronary artery disease, exercise training, $\mathrm{VO}_{2}$ peak response 


\section{Background}

Cardiac rehabilitation, including exercise training (ET), improves quality of life, morbidity and mortality in coronary artery disease (CAD) and has gained a class 1 , level of evidence $A$ recommendation (1). A $1 \mathrm{ml} / \mathrm{kg} / \mathrm{min}$ higher peak oxygen uptake $\left(\mathrm{VO}_{2}\right.$ peak) has been associated with a $15 \%$ decrease in all-cause and cardiovascular mortality in CAD (2). Unfortunately, $21-23 \%$ of patients fail to show a favourable $\mathrm{VO}_{2}$ peak response to training (35). Apart from genetics, sex, age and comorbidities, baseline physical fitness, physical activity and exercise dose are at play in the general population, but this has not been studied extensively in CAD (6).

\section{Aims}

We assessed whether cardiovascular risk factors, cardiopulmonary exercise test (CPET) variables, training intensity and physical activity can predict the $\mathrm{VO}_{2}$ peak response to exercise training in CAD patients. Early identification of $\mathrm{VO}_{2}$ peak non-responders could assist in personalisation and optimisation of exercise prescription.

\section{Methods}

We performed a subanalysis of the Study on Aerobic INTerval EXercise training in CAD patients (SAINTEX-CAD). In SAINTEX-CAD, patients with normal left ventricular ejection fraction and either stable CAD (after elective percutaneous coronary intervention ( $P C I$ ), conservative treatment, or coronary artery bypass graft (CABG)) or acute coronary syndrome (after primary $\mathrm{PCl}$ or $\mathrm{CABG}$ ) were randomised to 12 weeks of aerobic interval training (AIT) or aerobic continuous training (ACT) (7). We studied 167 of 200 patients, in whom VO 2 peak after 12 weeks was available. Non-response was defined as a change in $\mathrm{VO}_{2}$ peak $\left(\Delta \mathrm{VO}_{2}\right.$ peak) of $<1 \mathrm{ml} / \mathrm{kg} / \mathrm{min}$ (2). Maximal CPET was performed on a bicycle ergometer (Ergoline Schiller). Training intensity was defined as percentage of average heart rate over all sessions divided by baseline peak heart rate. Training adherence was recorded as number of training sessions completed. Physical activity during the first training week was evaluated in 75 patients using SenseWear Pro3 ArmbandTM (BodyMedia) (8).

Statistical analyses were performed using SPSS v24.0 (IBM). Baseline characteristics were analysed with independent samples T-test (normally distributed variables), Mann-Whitney U test (skewed variables) and chi-square test (categorical variables). Pearson (normally distributed variables) and Spearman (skewed variables) correlation coefficients were calculated between baseline variables and $\Delta \mathrm{VO}_{2}$ peak. Multiple linear regression models ( $\triangle \mathrm{VO}_{2}$ peak, method 'backward', variables in 1 block: sex, age, elective $\mathrm{PCl}$, oxygen uptake efficiency slope (OUES), training intensity, steps $/ 12 \mathrm{~h}$, energy expenditure/12h and sedentary time/12h) and multiple logistic regression models (responder status, method 'enter', variables in 1 block: age, elective $\mathrm{PCl}$ and baseline $\mathrm{VO}_{2}$ peak) were used to assess independent determinants of training response.

\section{Results}

Of 167 included patients (155 males), 24 were $\mathrm{VO}_{2}$ peak non-responders (14\%, 22 males, 46\% AIT). 
Baseline characteristics according to responder status are shown in Table 1. Pharmacological therapy was similar between groups (all $p>0.05$, data not shown). Non-responders were older patients $(p=0.009)$, more frequently included after elective $\mathrm{PCl}(p=0.007)$ and had a lower prevalence of diabetes mellitus $(p=0.029)$. Non-responders had higher baseline $\mathrm{VO}_{2}$ peak, OUES, \% predicted $\mathrm{VO}_{2}$ peak and \% predicted workload compared to responders (all $p<0.05$ ). Actual training intensity and training adherence were similar between responders and non-responders. Non-responders showed a trend towards higher sedentary time, less energy expenditure and fewer steps $(/ 12 h$, all $p>0.05)$.

In a multivariate analysis (Table 2), age, baseline $\mathrm{VO}_{2}$ peak, \%predicted $\mathrm{VO}_{2}$ peak, \%predicted workload and OUES were negatively correlated with $\triangle \mathrm{VO}_{2}$ peak, whereas training intensity was positively correlated with $\Delta \mathrm{VO}_{2}$ peak. Again, $\Delta \mathrm{VO}_{2}$ peak was related to baseline physical activity, with lower energy expenditure and steps/12h, and higher sedentary time/12h resulting in a lower $\Delta \mathrm{VO}_{2}$ peak.

In a linear regression analysis, older age, history of elective $\mathrm{PCl}$, higher OUES, lower training intensity and lower baseline energy expenditure were independent predictors of a lower $\Delta \mathrm{VO}_{2}$ peak (Table 2). This model explained $41 \%$ of the variability in $\Delta \mathrm{VO}_{2}$ peak. In logistic regression analyses older age, history of elective $\mathrm{PCl}$ and higher baseline $\mathrm{VO}_{2}$ peak were independent predictors of non-response to exercise training (Table 2).

\section{Conclusion}

In this subanalysis of SAINTEX-CAD, 24 out of 167 CAD patients (14\%) were $\mathrm{VO}_{2}$ peak nonresponders, equally distributed across both training regimes. Older age, inclusion after elective $\mathrm{PCl}$, higher baseline $\mathrm{VO}_{2}$ peak and OUES, lower training intensity and lower baseline energy expenditure were predictors of impaired $\mathrm{VO}_{2}$ peak trainability. Older age, inclusion after elective $\mathrm{PCl}$, higher baseline OUES and lower training intensity and baseline energy expenditure explained $41 \%$ of the variability in $\Delta \mathrm{VO}_{2}$ peak. By carefully assessing these variables upon inclusion for cardiac rehabilitation, ENR can be identified early and this could be a first step towards patient tailored exercise prescription.

Older age, higher baseline exercise performance, lower training frequency and intensity have been described as predictors of training non-response $(4,9)$. Patients who underwent $\mathrm{PCl}$ had a lower improvement in $\mathrm{VO}_{2}$ peak compared to CABG or AMI, which was partly explained by the higher baseline exercise performance in these patients (9). In the present study, non-responders were possibly more physically active prior to engaging in CR. However, this could not be confirmed by physical activity state prior to rehabilitation. Possibly, non-responders had a higher baseline $\mathrm{VO}_{2}$ peak due to better physical fitness, despite their sedentary state.

Patients that already had a high OUES at baseline, showed less improvement in $\mathrm{VO}_{2}$ peak following 3 months of exercise training. OUES is strongly related to $\mathrm{VO}_{2}$ peak (10) and is an independent predictor of all-cause and cardiovascular mortality in CAD patients (11).

Furthermore, a high $\mathrm{VO}_{2}$ peak at baseline also resulted in a lower increase in $\mathrm{VO}_{2}$ peak, which is consistent with previous studies $(5,12,13)$. Whether increasing training duration, intensity or frequency can improve the $\mathrm{VO}_{2}$ peak response in these patients remains to be determined in larger trials. 
Table 1: Univariate analysis: difference between non-responders and responders

\begin{tabular}{|c|c|c|c|c|}
\hline & Baseline parameters & Non-responders $n=24$ & Responders $n=143$ & $\mathbf{p}$ \\
\hline \multirow[t]{9}{*}{ CV risk factors } & Age (years) & $63.1( \pm 8.1)$ & $58.0( \pm 8.9)$ & 0.009 \\
\hline & Sex & 22 men $(92 \%)$ & 133 men (93\%) & 0.684 \\
\hline & $\mathrm{BMI}\left(\mathrm{kg} / \mathrm{m}^{2}\right)$ & $26.7(22.3-39.9)$ & $28.0(19.8-36.7)$ & 0.418 \\
\hline & History of hypertension & $11(46 \%)$ & $77(54 \%)$ & 0.467 \\
\hline & History of diabetes mellitus & $1(4 \%)$ & $34(24 \%)$ & 0.029 \\
\hline & Total cholesterol & $152.9( \pm 27.6)$ & $135.7( \pm 27.4)$ & 0.005 \\
\hline & LDL cholesterol & $83.4( \pm 23.6)$ & $71.8( \pm 21.2)$ & 0.015 \\
\hline & HDL cholesterol & $49(27-67)$ & $42(18-85)$ & 0.073 \\
\hline & Triglycerides & $104(53-562)$ & $105(51-567)$ & 0.769 \\
\hline \multirow[t]{5}{*}{ Type of CAD } & Stable CAD post-CABG & $3(13 \%)$ & $44(31 \%)$ & 0.066 \\
\hline & Stable CAD elective $\mathrm{PCl}$ & $8(33 \%)$ & $15(10 \%)$ & 0.007 \\
\hline & ACS conservative & 0 & $7(5 \%)$ & 0.595 \\
\hline & ACS CABG & 0 & $3(2 \%)$ & 1.0 \\
\hline & ACS primary $\mathrm{PCl}$ & $13(54 \%)$ & $74(52 \%)$ & 0.826 \\
\hline \multirow[t]{11}{*}{ CPET } & Resting HR (bpm) & $61(49-76)$ & $65(37-100)$ & 0.090 \\
\hline & $\mathrm{VO}_{2}$ peak (I/min) & $2.05(1.1-3.5)$ & $1.89(0.9-3.4)$ & 0.044 \\
\hline & $\mathrm{VO}_{2}$ peak $(\mathrm{ml} / \mathrm{kg} / \mathrm{min})$ & $25.2(16.8-46.2)$ & $22.1(9.8-33.5)$ & 0.042 \\
\hline & $\%$ predicted $\mathrm{VO}_{2}$ peak (\%) & $102.8( \pm 26.9)$ & $81.3( \pm 19.4)$ & 0.001 \\
\hline & $\begin{array}{l}\text { Work economy } \\
\text { (watt } / \mathrm{ml} / \mathrm{kg} / \mathrm{min} \text { ) }\end{array}$ & $0.30(0.21-0.51)$ & $0.28(0.14-0.45)$ & 0.083 \\
\hline & Resting systolic BP (mmHg) & $123( \pm 18.5)$ & $119( \pm 16.5)$ & 0.339 \\
\hline & Peak systolic BP (mmHg) & $182( \pm 30.5)$ & $172( \pm 28.6)$ & 0.111 \\
\hline & Peak work load (Watt) & $160(60-240)$ & $140(60-260)$ & 0.246 \\
\hline & $\%$ predicted work load (\%) & $124.4(59.4-180.3)$ & $96.4(32.0-179.5)$ & 0.007 \\
\hline & OUES & $2106.1(849.7-3910.4)$ & $1873.8(920.1-3615.3)$ & 0.020 \\
\hline & VE/VCO2 slope & $31.1(20.3-47.3)$ & $29.9(17.0-49.6)$ & 0.475 \\
\hline \multirow[t]{3}{*}{ Training } & $\begin{array}{l}\text { Group assignment AIT/ACT (n, } \\
\%)\end{array}$ & $\begin{array}{l}\text { AIT } 11(46 \%) \\
\text { ACT } 13(54 \%)\end{array}$ & $\begin{array}{l}\text { AIT } 71(50 \%) \\
\text { ACT } 72(50 \%)\end{array}$ & 0.729 \\
\hline & Training intensity (\%) & $79.9( \pm 9.5)$ & $84.8( \pm 9.6)$ & 0.104 \\
\hline & $\begin{array}{l}\text { Adherence (number of training } \\
\text { sessions) }\end{array}$ & $36(32-36)$ & $36(30-42)$ & 0.224 \\
\hline \multirow{5}{*}{$\begin{array}{l}\text { Physical } \\
\text { activity }\end{array}$} & & $\mathrm{n}=12$ & $n=63$ & \\
\hline & On-body time $(\mathrm{h})$ & $69.5(64.8-81.9)$ & $70.4(34.6-117.5)$ & 0.902 \\
\hline & Sedentary time per $12 \mathrm{~h}$ & $10.7(9.0-11.4)$ & $10.2(6.4-11.7)$ & 0.059 \\
\hline & $\begin{array}{l}\text { Total energy expenditure (on- } \\
\text { body, per } 12 \mathrm{~h} \text { ) }\end{array}$ & $1528.7(1118.1-2213.1)$ & $\begin{array}{l}1688.0(1245.0- \\
3552.4) \\
\end{array}$ & 0.071 \\
\hline & Total number of steps per $12 \mathrm{~h}$ & $4898.6( \pm 1669.3)$ & $6081.9( \pm 2213.7)$ & 0.083 \\
\hline
\end{tabular}

$\mathrm{ACS}=$ acute coronary syndrome, $\mathrm{ACT}=$ aerobic continuous training, $\mathrm{AIT}=$ aerobic interval training, $\mathrm{BMI}=$ body mass index, $\mathrm{BP}=$ blood pressure, $\mathrm{CABG}=$ coronary artery bypass graft, $\mathrm{CAD}=$ coronary artery disease, $\mathrm{CPET}=$ cardiopulmonary exercise test, $\mathrm{CV}=$ cardiovascular, $\mathrm{HR}=$ heart rate, OUES= oxygen uptake efficiency slope, $\mathrm{PCl}=$ percutaneous coronary intervention. 
Table 2: Multivariate analysis: Association of change in $\mathrm{VO}_{2}$ peak with baseline variables

\begin{tabular}{|c|c|c|c|c|c|c|c|}
\hline & \multirow{2}{*}{$\begin{array}{l}\text { Baseline } \\
\text { parameters }\end{array}$} & \multicolumn{2}{|c|}{ Correlation } & \multicolumn{2}{|c|}{ Linear regression } & \multicolumn{2}{|c|}{ Logistic regression } \\
\hline & & $r$ & $\mathbf{p}$ & B $(95 \% \mathrm{Cl})$ & $\mathbf{p}$ & $\begin{array}{l}\text { Odds ratio } \\
(95 \% \mathrm{Cl})\end{array}$ & $\mathbf{p}$ \\
\hline $\begin{array}{l}\text { CV risk } \\
\text { factors }\end{array}$ & Age (years)* & -0.238 & 0.002 & $\begin{array}{l}-0.605 \\
(-0.94--0.27)\end{array}$ & 0.001 & $\begin{array}{l}1.11 \\
(1.04-1.18)\end{array}$ & 0.001 \\
\hline $\begin{array}{l}\text { Type of } \\
\text { CAD }\end{array}$ & Elective $\mathrm{PCl}$ & - & - & $\begin{array}{l}-15.401 \\
(-26.96--3.84)\end{array}$ & 0.010 & $\begin{array}{l}3.31 \\
(1.12-9.76)\end{array}$ & 0.030 \\
\hline \multirow[t]{4}{*}{ CPET } & $\begin{array}{l}\mathrm{VO}_{2} \text { peak } \\
(\mathrm{mL} / \mathrm{kg} / \mathrm{min})^{*}\end{array}$ & -0.238 & 0.002 & - & - & $\begin{array}{l}1.16 \\
(1.06-1.27)\end{array}$ & 0.001 \\
\hline & $\begin{array}{l}\text { \% Predicted } \\
\mathrm{VO}_{2} \text { peak } \\
(\mathrm{mL} / \mathrm{kg} / \mathrm{min})^{*}\end{array}$ & -0.380 & $<0.001$ & - & - & - & - \\
\hline & $\begin{array}{l}\text { \%Predicted } \\
\text { workload (W)* }\end{array}$ & -0.292 & $<0.001$ & - & - & - & - \\
\hline & OUES** & -0.182 & 0.019 & $\begin{array}{l}-0.014 \\
(-0.02--0.01)\end{array}$ & $<0.001$ & - & - \\
\hline Training & $\begin{array}{l}\text { Training intensity } \\
(\%)^{*}\end{array}$ & 0.315 & 0.004 & $\begin{array}{l}0.447 \\
(0.12-0.77) \\
\end{array}$ & 0.008 & - & - \\
\hline \multirow[t]{3}{*}{$\begin{array}{l}\text { Physical } \\
\text { activity }\end{array}$} & $\begin{array}{l}\text { Baseline energy } \\
\text { expenditure/12h } \\
\text { (kcal/12h)* }\end{array}$ & 0.261 & 0.024 & $\begin{array}{l}0.014 \\
(0.01-0.02)\end{array}$ & 0.003 & - & - \\
\hline & Steps/12h* & 0.329 & 0.004 & - & - & - & - \\
\hline & $\begin{array}{l}\text { Sedentary } \\
\text { time/12h** }\end{array}$ & -0.235 & 0.043 & - & - & - & - \\
\hline
\end{tabular}

*Pearson or **Spearman correlation. $\mathrm{CAD}=$ coronary artery disease, $\mathrm{Cl}=$ confidence interval, $\mathrm{CPET}=$ cardiopulmonary exercise test, $\mathrm{CV}=$ cardiovascular, OUES= oxygen uptake efficiency slope, $\mathrm{PCl}=$ percutaneous coronary intervention, $\mathrm{VO}_{2}$ peak = peak oxygen uptake.

\section{References}

1. Roffi M, Patrono C, Collet J-P, Mueller C, Valgimigli M, Andreotti F, et al. 2015 ESC Guidelines for the management of acute coronary syndromes in patients presenting without persistent ST-segment elevation. European Heart Journal. 2016; 37(3):267315.

2. Keteyian SJ, Brawner CA, Savage PD, Ehrman JK, Schairer J, Divine G, et al. Peak aerobic capacity predicts prognosis in patients with coronary heart disease. American Heart Journal. 2008; 156(2):292-300.

3. Timmons JA, Knudsen S, Rankinen T, Koch LG, Sarzynski M, Jensen T, et al. Using molecular classification to predict gains in maximal aerobic capacity following endurance exercise training in humans. Journal of Applied Physiology. 2010; 108(6):1487-96. 
4. De Schutter A, Kachur S, Lavie CJ, Menezes A, Shum KK, Bangalore S, et al. Cardiac Rehabilitation Fitness Changes and Subsequent Survival. Eur Heart J Qual Care Clin Outcomes. 2018; 4(3):173-9.

5. Savage PD, Antkowiak M, Ades PA. Failure to Improve Cardiopulmonary Fitness in Cardiac Rehabilitation. Journal of Cardiopulmonary Rehabilitation. 2009; 29:284-91.

6. Bouchard C, An P, Rice T, Skinner JS, Wilmore JH, Gagnon J, et al. Familial aggregation of VO2max response to exercise training: results from the HERITAGE Family Study. Journal of Applied Physiology. 1999; 87:1003-8.

7. Conraads VM, Van Craenenbroeck EM, Pattyn N, Cornelissen VA, Beckers PJ, Coeckelberghs $\mathrm{E}$, et al. International Journal of Cardiology. International Journal of Cardiology. 2013; 168(4):3532-6.

8. Scheers T, Philippaerts R, Lefevre J. Variability in physical activity patterns as measured by the SenseWear Armband: how many days are needed? European Journal of Applied Physiology. 2011; 112(5):1653-62.

9. Vanhees L, Stevens A, Schepers D, Defoor J, Rademakers F, Fagard R. Determinants of the effects of physical training and of the complications requiring resuscitation during exercise in patients with cardiovascular disease. European Journal of Cardiovascular Prevention \& Rehabilitation. 2004; 11(4):304-12.

10. Van de Veire NR, Van Laethem C, Philippé J, De Winter O, De Backer G, Vanderheyden $\mathrm{M}$, et al. $\mathrm{VE} / \mathrm{VCO}_{2}$ slope and oxygen uptake efficiency slope in patients with coronary artery disease and intermediate peakVo $\mathrm{O}_{2}$. European Journal of Cardiovascular Prevention \& Rehabilitation. 2006; 13(6):1-8.

11. Coeckelberghs E, Buys R, Goetschalckx K, Cornelissen VA, Vanhees L. Prognostic value of the oxygen uptake efficiency slope and other exercise variables in patients with coronary artery disease. European Journal of Preventive Cardiology. 2015; 23(3):23744.

12. Shiran A, Kornfeld S, Zur S, Laor A, Karelitz Y, Militianu A, et al. Determinants of Improvement in Exercise Capacity in Patients Undergoing Cardiac Rehabilitation. Cardiology. 1997; 88:207-13.

13. Lavie CJ, Milani RV. Patients with high baseline exercise capacity benefit from cardiac rehabilitation and exercise training programs. American Heart Journal. 1994; 128(6):1105-9. 